\section{Psychological and psychopathological sequelae in cardiovascular acute disease}

\section{Pasquale Caponnetto}

Center for Prevention and Care of the Tabagism, University-Hospital "Policlinico

V. Emanuele", University of Catania,

Italy; Department of Clinical and

Experimental Biomedicine, University of

Catania, Italy; Institute for Social

Marketing, University of Stirling, UK

\section{Abstract}

The burden of mental illness is profound and growing. Each year, almost one in three adults in the non-institutionalized community has a diagnosable mental or addictive disorder, and this figure climbs to approximately $40 \%$ among emergency departments patients. We described the principal cardiovascular acute disease and their emotional and behavioral consequences where psychological intervention could improve the care pathway and clinical outcome. Peer-reviewed articles from Medline, Psycinfo, Web of Science, Scopus, and Cochrane library, about psychological and psychopathological sequelae in cardiovascular acute disease were searched. The psychological and psychopathological sequelae associated to stroke include emotional and behavioral changes and cognitive impairment. Fear, symptoms of depression, anxiety or specific post-traumatic symptoms like intrusions, hyper-arousal and/or cognitive avoidance are common in people suffering of cardiovascular acute disease treated at emergency departments. In emergency departments, health personnel must recognize psychological and psychopathological sequelae in cardiovascular acute disease in order to develop effective interventions for these patients. Identify factors that are associated with both psychological distress and physical distress and promote interventions aimed at reducing psychological distress and improving psychological health empowerment is an important element to consider in order to offer the best care to vulnerable population as that suffering of cardiovascular acute disease.

\section{Introduction}

The burden of mental illness is profound and growing. ${ }^{1}$ Each year, almost one in three adults in the non-institutionalized community has a diagnosable mental or addictive disorder, and this figure climbs to approximately $40 \%$ among emergency departments (ED) patients. ${ }^{1}$ Mental disorders are the fastest growing component of emergency medical practice. ${ }^{1}$ Persons, who need psychiatric care also need general medical care, and those with severe mental disorders suffer excess morbidity and mortality compared with the general population. ${ }^{2}$ Indeed, from substance abuse to suicide attempts, ED has long provided expertise in the art and science of clinical toxicology and acute psychiatric and psychological stabilization ${ }^{3}$ For these reasons, emergency physicians (EP) and emergency nurses (EN) must increase their psychosocial research armamentarium if they are to meaningfully improve emergency departments clinical practice.

\section{Materials and Methods}

The aim of this mini review was to evaluate the state-of-the-science focused on psychological and psychopathological sequelae in cardiovascular acute disease. A search on psychological and psychopathological sequelae in cardiovascular acute disease was conducted. The review search period was from January 2000 - November 2017. The search was not restricted by language or geographical region. Databases searched included MEDLINE, PsycINFO, Web of Science, Scopus, and Cochrane Library. Additionally, reference lists of all included papers were checked for any citations missed by electronic database searching. Cohort and case-control study designs were considered eligible for inclusion. Cross-sectional studies, case series and case reports were included.

\section{Discussion}

\section{Strategies for emergency manage- ment}

In emergency management, individuals must work together to contain and resolve a hazardous event. With each team member performing a specialized function, the effectiveness of the team depends upon the ability of its members to communicate with each other to coordinate activities, to share information, and to implement appropriate strategies. ${ }^{4}$ The diagnosis of a severe disease with subsequent intensive care signifies exposure to psychological and physical stress could constitutes a traumatic experience for most people. As a result of this experience, many people suffer from clini-
Correspondence: Pasquale Caponnetto, Department of Educational Sciences, Università degli Studi di Catania, Piazza Università 2, 95131 Catania, Italy.

E-mail: pcapon@unict.it

Key words: mental disorders; cardiovascula acute disease; psychological and psychopathological sequelae.

Conflict of interest: the author declares no conflict of interest.

Funding: none.

Received for publication: 26 September 2018. Revision received: 28 October 2018.

Accepted for publication: 29 October 2018.

This work is licensed under a Creative Commons Attribution-NonCommercial 4.0 International License (CC BY-NC 4.0).

CC Copyright P. Caponnetto , 2018

Licensee PAGEPress, Italy

Mental Illness 2018; 10:7887

doi:10.4081/mi.2018.7887

cally relevant symptoms of depression, anxiety or specific post-traumatic symptoms like intrusions, hyper-arousal and/or cognitive avoidance. 5 The underlying psychosocial processes in the after math of the confrontation with the diagnosis and ensuing intensive medical treatment have been variously referred in scientific literature, as post-traumatic growth (PTG), stress-related growth, adversarial growth. 5 With this in mind, we would describe the principal cardiovascular acute disease and their emotional and behavioral consequences, in which a psychological intervention could improve the care pathway and clinical outcome (Figure 1 and Table 1).

\section{The psychological and psychopatho- logical sequelae}

In the UK around 150.000 people have a stroke each year and stroke is credited with having a greater disability impact than any other chronic disease. The psychological and psychopathological sequelae associated to stroke include emotional and behavioral changes and cognitive impairment. Recently there has been increased recognition of these problems and directives to improve services that manage them (Figure 2). ${ }^{6}$ Identifying emotional problems after stroke can be complicated by the overlap between the symptoms of stroke and symptoms of mental health disorders, as well as by impairment in cognitive and communication ability. Depressive disorders following stroke are common. Estimates of the 
frequency range from $25 \%$ to $79 \%$, with most studies indicating the rate being approximately $30 \% .{ }^{7}$ However, the consistent finding is that many people have low mood, which may require treatment. Antidepressant medication is often used, but concomitant drug therapies and adverse effects may also limit the extent to which antidepressant drugs are appropriate. ${ }^{7}$ From this point of view, Cognitive Behavioral Therapy (CBT) could represent a possible psychological approach. However, CBT in the treatment of depression following stroke was found to be ineffective in some randomized clinical trials. 8,9

With regard to the acute myocardial infarction (AMI), a number of previous studies addressed the effect of psychological interventions in these patients, but it is not known whether psychotherapy might be beneficial after medical and interventional therapy of AMI. Results from a randomized, controlled study to assess the effects of a short-term psychotherapy (STP) on the clinical outcomes of patients who underwent an emergency percutaneous coronary intervention after AMI have shown a clinical improvement. Specifically, adding STP to cardiological therapy improves cardiological symptoms, quality of life, and psychological and medical outcomes one year after AMI, while reducing the need for rehospitalizations. ${ }^{10}$

Studies focusing on the effects of antidepressant treatment in cardiac patients have found modest effects on depressive symptoms but not on cardiac outcomes. ${ }^{11}$ In analysis of data from the Enhancing Recovery in Coronary Heart Disease trial, the researchers assessed whether somatic and cognitive depressive symptoms improved after cognitive behavior therapy (CBT) and whether changes in somatic or cognitive depressive symptoms following acute myocardial infarction (MI) were related to event-free survival. They also assessed whether these associations differed by treatment arm since an earlier study based on the ENRICHD trial showed that intervention patients whose depression did not improve were at higher risk for late mortality than were patients who responded to treatment. ${ }^{12}$ The authors found that the positive changes in somatic depressive symptoms, and not cognitive depressive symptoms, were related to event-free survival after treatment with CBT in depressed patients with MI. This association was independent of demographic and clinical variables, including disease severity, and was restricted to the intervention arm. ${ }^{12}$

Also, Post-traumatic Stress Disorder (PTSD) may occur after the experience of a traumatic event. Life-threatening illnesses, such as myocardial infarction, can cause a PTSD. 13 Several socio-demographic and psychosocial risk factors for the risk of MItriggered PTSD have been identified, including young age and increased distress perceived during MI. PTSD is associated with impaired quality of life, social functioning, and high economic burden to the society. PTSD and posttraumatic stress along a continuum of severity are predictive of incident cardiovascular disease morbidity and mortality.

Many patients who attend an emergency department (ED) with chest pain receive a diagnosis of non-cardiac chest pain (NCCP), and often suffer of psychological distress and continued chedpain. ${ }^{14}$ Acute chest pain accounts for approximately 700,000 emergency department (ED) attendances each year in England and Wales, but between 30 and $60 \%$ of these patients do not receive a cardiac diagnosis for their pain. ${ }^{15}$ In order to develop effective interventions for this population, it is important to identify factors that are associated with both psychological distress and continued chest pain. However, research to date has focused on a limited range of mainly demographic variables and has lacked a strong theoretical focus. Some authors suggest that continued chest pain is related to psychological distress and poor quality of life (QoL), for this reason, interventions should be aimed at reducing psychological distress and improving QoL. According their point of view, given the associations between perceived psychological causes and psychological distress/QoL, NCCP patients in the ED might benefit from psychological therapies to manage their chest pain. ${ }^{14}$

Table 1. CVD disease, psychological/psychopathological effect and suggested treatment.

\begin{tabular}{lcc} 
Disease & Psychological/psychopathological effect & Treatment \\
Stroke & Depressive disorders & Cognitive impairment \\
Acute myocardial infarction (AMI) & Depression, anxiety or Post-traumatic & Antidepressant medication plus CBT \\
& Stress Disorder (PTSD) & (Cognitive Behavioral Therapy) \\
\hline Acute chest pain & Psychological distress and poor quality of life (QoL) & Interventions reducing psychological distress and improving QoL \\
\hline
\end{tabular}

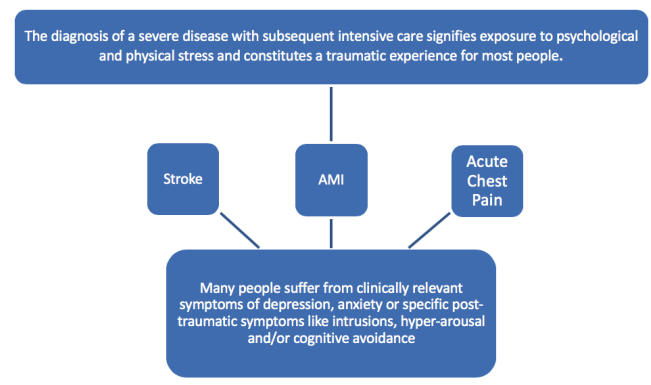

Figure 1. Diagnosis of a severe CVD disease and psychological effects.

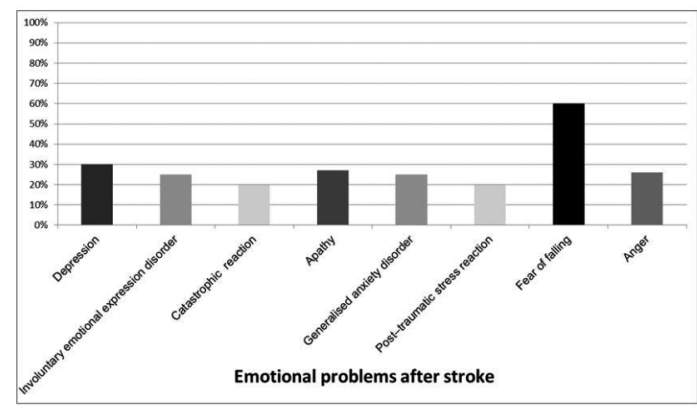

Figure 2. Emotional problems after stroke 


\section{Conclusions}

In order to develop effective interventions for patients with cardiovascular acute disease, it is important to identify factors that are associated with both psychological distress and continued pain and promote interventions aimed at reducing psychological distress and improving quality of life such as short-term psychotherapy (STP) and Cognitive behavior therapy (CBT).

Hence, new psychological perspective to cardiovascular acute disease is crucial. It is crystal clear that actually one of the main aims of health psychology is the prevention and support of psychological disease, that results in decreasing costs and improving health and quality of life. Hence, by knowing the psychological risk elements and protective components of coronary artery disease, prevention, control and health empowerment can be performed. These activities result in a decrease in risk factors, decrease in treatment costs, improvement in quality of life quality, and decrease in illnesses.

\section{References}

1. Larkin GL, Beautrais AL, Milzman DP. Mental Health and Emergency Medicine: A Research Agenda. Acad Emerg Med 2009;16:1110-9.

2. Griswold KS, Servoss TJ, Leonard KE et al. Connections to Primary Medical Care after Psychiatric Crisis. J Am Board Fam Pract 2005;18:166-72.

3. Hughes AA, Bogdan GM, Dart RC, et al. Active surveillance of abused and misused prescription opioids using poison center data: a pilot study and descriptive comparison. Clin Toxicol (Phila) 2007;45:144-51.

4. Dunn JC, Lewandowsky S, Kirsner K. Dynamics of Communication in Emergency Management. Appl Cognit Psychol 2002;16:719-37.

5. Barskova T, Oesterreich R. Post-traumatic growth in people living with a serious medical condition and its relations to physical and mental health: A systematic review. Disabil Rehabil 2009;31:1709-33.

6. Kneebone II, Lincoln NB. Psychological Problems after Stroke and Their Management: State of Knowledge. Neuros Med 2012;3:83-9.

7. Meijer A, Conradi HJ, Bos EH, et al. Prognostic association of depression following myocardial infarction with mortality and cardiovascular events: a meta-analysis of 25 years of research. Gen Hosp Psych 2011;33:203-16.

8. Lincoln NB, Flannaghan T. Cognitive Behavioral Psychotherapy for Depression Following Stroke: A Randomized Controlled Trial. Stroke 2003;34:111-5.

9. Hackett ML, Anderson CS, House AO, et al. Interventions for Treating Depression after Stroke. Stroke 2009;40:487-8.

10. Roncella A, Giornetti A, Cianfrocca C, et al. Rationale and trial design of a randomized, controlled study on short-term psychotherapy after acute myocardial infarction: the STEP-IN-AMI trial
(Short Term Psychotherapy in Acute Myocardial Infarction). J Cardiovasc Med (Hagerstown) 2009;10:947-52.

11. van Melle JP, de Jonge P, Honig A, et al. MIND-IT investigators. Effects of antidepressant treatment following myocardial infarction. $\mathrm{Br} \mathrm{J}$ Psychiatry 2007;190:460-6

12. Roesta AE, Carney RM., Freedland Kenneth E, et al. Changes in cognitive versus somatic symptoms of depression and event-free survival following acute myocardial infarction in the Enhancing Recovery In Coronary Heart Disease (ENRICHD) study. J Affect Disord 2013;149:335-41.

13. Meister R, Princip M, Schmid JP, et al. Myocardial Infarction - Stress Prevention INTervention (MI-SPRINT) to reduce the incidence of posttraumatic stress after acute myocardial infarction through trauma-focused psychological counseling: study protocol for a randomized controlled trial. Trials 2013;14:329.

14. Webstera R, Normana P, Goodacreb S, et al. Illness representations, psychological distress and non-cardiac chest pain in patients attending an emergency department. Psychol Health 2014;29: 1265-82.

15. Eken C, Oktay C, Bacanli A, et al. Anxiety and depressive disorders in patients presenting with chest pain to the emergency department: A comparison between cardiac and non-cardiac origin. J Emerg Med 2010;39:144-50. 